\title{
Retropharyngeal lymph node-sparing radiotherapy in patients with oropharyngeal carcinoma
}

\author{
Byung-Hee Kang', Keun-Yong Eom², Changhoon Song², Jin Ho Kim', Hong-Gyun Wu', In Ah Kim², Jae-Sung Kim² \\ ${ }^{1}$ Department of Radiation Oncology, Seoul National University College of Medicine, Seoul, Korea \\ ${ }^{2}$ Department of Radiation Oncology, Seoul National University College of Medicine, Seoul National University Bundang Hospital, Seongnam, \\ Korea
}

Received: March 24, 2021

Revised: May 20, 2021

Accepted: May 28, 2021

Correspondence:

Keun-Yong Eom

Department of Radiation Oncology, Seoul National University Bundang Hospital, 82 Gumi-ro 173beon-gil, Bundang-gu, Seongnam 13620,

Korea

Tel: +82-31-787-7653

E-mail: 978sarang@snubh.org

ORCID:

https://orcid.org/0000-0003-3650-1133
Purpose: In radiotherapy for head and neck cancer, it is crucial to define the appropriate treatment volume to determine treatment outcome and toxicity. We examined the feasibility of omitting elective high retropharyngeal lymph node (RPLN) irradiation in patients with oropharyngeal cancer.

Materials and Methods: We performed a retrospective review of 189 patients with oropharyngeal squamous cell carcinoma who were treated with definitive or postoperative radiation therapy between 2009 and 2016. Of them, 144 (76.2\%) underwent ipsilateral RPLN irradiation up to the superior border of the $\mathrm{C} 1$ vertebral body, while the other $45(23.8 \%)$ were irradiated up to the transverse process of the $\mathrm{C} 1$ vertebra. High RPLN-treated and spared group were propensity matched based on key clinical variables.

Results: During the follow-up period, only three patients (one in the high RPLN-treated group and two in the high RPLN-spared group) developed RPLN recurrence. There were no significant between-group differences in 5-year locoregional failure-free survival ( $82.8 \%$ vs. 90.6\%; $p=0.14$ ), distant metastasis-free survival (93.1\% vs. 93.3\%; $p=0.98)$ and RPLN failure-free survival ( $99.3 \%$ vs. $95.0 \% ; p=0.09$ ). In the matched groups, high RPLN-spared patients received a lower mean ipsilateral parotid gland dose (mean, 20.8 Gy vs. $29.9 \mathrm{~Gy} ; \mathrm{p}<0.001$ ) and had a lower incidence of chronic xerostomia (grade $0,43.5 \%$ vs. $13.0 \% ; p=0.023$ ) at 1 year after radiotherapy compared with high RPLN-treated patients.

Conclusion: Omission of ipsilateral high RPLN irradiation seems safe, and reduces the incidence of chronic xerostomia in patients with oropharyngeal squamous cell carcinoma.

Keywords: Oropharyngeal cancer, Retrophagyngeal lymph node, Locoregional recurrence, Xerostomia

\section{Introduction}

The incidence of tobacco-associated head and neck squamous cell carcinoma has steadily declined over the past few decades, whereas the incidence of human papillomavirus (HPV)-induced oropharyngeal cancer (OPC) has been increasing [1-3]. Patients with HPV-induced OPC are younger and have fewer comorbidities and a more favorable prognosis than those with tobacco-associated squamous cell carcinoma [4-6]. While cisplatin-based concurrent chemoradiotherapy (CCRT) is the standard treatment for OPC, some patients who present at an early stage are candidates for primary surgery [7]. Patients with HPV-induced OPC show a good response to CCRT, and the 5 -year overall survival is approximately $80 \%$ to 90\% [8]. However, the treatment-related morbidity is considerable. Almost all patients suffer from acute oral mucositis, chronic xerostomia, and loss of taste [9]. Since patients with HPV-induced OPC are generally young and likely to survive their disease, treatment-related toxicity is concerning [10-12]. 
Intensity-modulated radiation therapy allows conformal dose distribution around the tumor and organs-at-risk. Delineation of the clinical target volume (CTV) and organs-at-risk is very important because treatment outcomes and toxicities depend on treatment plan. However, physicians often disagree about the optimal target definition. In nasopharyngeal, oropharyngeal, and hypopharyngeal cancers, there is a risk of metastasis to the retropharyngeal lymph nodes (RPLNs) [13]. Since high RPLNs are located adjacent to the parotid glands and pharyngeal constrictor muscles, irradiation of these regions has been shown to compromise the quality of life $(\mathrm{OOL})$ of patients with head and neck cancer $[14,15]$. According to consensus guidelines for the delineation of neck node levels, in patients with primary pharyngeal involvement, the bilateral RPLNs should be treated up to the upper edge of the $\mathrm{C} 1$ vertebral body/ hard palate cranially [13]. However, previous studies indicate that sparing the contralateral high RPLN is associated with minimal risk of failure and improves QOL [16-18]. Kjems et al. [19] argued that ipsilateral RPLN sparing is safe in patients with OPC in whom the posterior pharyngeal wall is not involved. In this study, we aimed to evaluate the safety and feasibility of omitting ipsilateral high RPLN irradiation in patients with OPC. Background and purpose should be stated clearly.

\section{Materials and Methods}

\section{Patient selection}

The Institutional Review Board of Seoul National University Hospital and Seoul National University Bundang Hospital approved this study (No. B-1805-471-402). The informed consent was waived. We retrospectively reviewed 189 patients with pathologically proven oropharyngeal squamous cell carcinoma who received radiotherapy (RT) at the aforementioned hospitals between January 2009 and December 2016. The inclusion criteria were age $>18$ years and the Eastern Cooperative Oncology Group performance status of $0-2$. The exclusion criteria were presence of distant metastasis or initial evidence of RPLN involvement.

\section{Treatment}

Of the 189 patients, 160 (84.7\%) received intensity-modulated radiation therapy, and 29 (15.3\%) received three-dimensional conformal radiotherapy. Definitive radiotherapy typically included a dose of 67.5-70 Gy to high-risk regions. Patients treated postoperatively or after induction chemotherapy received 60-63 Gy to high-risk regions. The doses for intermediate- and low-risk areas were 54-56 Gy and 42-48 Gy, respectively. Among all patients, 62 (32.8\%) received definitive CCRT, 53 (28.0\%) underwent induction chemotherapy followed by CCRT/RT alone, 60 (31.7\%) were offered surgery and postoperative CCRT/RT alone, 5 (2.6\%) underwent induction chemotherapy followed by surgery and postoperative CCRT/RT alone, and 9 (4.8\%) were treated with definitive RT alone. After radiotherapy, patients were evaluated at 2 weeks after treatment. They were initially followed up every 1-2 months, followed by every 3 months for a year, every 3-4 months for 2 years, and every 6 months thereafter. During follow-up, physical examination, nasopharyngeal laryngoscopy, and imaging studies including contrast-enhanced neck computed tomography and/or magnetic resonance imaging were performed. Symptoms related to xerostomia were evaluated using the Radiation Therapy Oncology Group and the European Organization for Research and Treatment of Cancer toxicity scale at each follow-up visit [20].

\section{RPLN evaluation}

To evaluate the coverage of the dose to the RPLN and parotid gland, we retrospectively reviewed each patient's treatment plan. According to consensus guidelines, the cranial border of the RPLN is the upper edge of the $\mathrm{C} 1$ vertebral body/hard palate [13]. Patients were classified into the high RPLN-treated group ( $n=144$ ) when the CTV sufficiently encompassed the RPLNs between the upper edge of the $\mathrm{C} 1$ vertebral body and the inferior border of the transverse process of the $\mathrm{C} 1$ vertebra. The remaining patients ( $\mathrm{n}=$ 45) were classified into the high RPLN-spared group.

\section{Statistical analysis}

To compare clinical variables according to high RPLN treatment status, Student t-test, Wilcoxon rank sum test, and chi-square test were used, as appropriate. To control for differences in characteristics between the two groups according to high RPLN treatment status, we conducted the propensity score matching analysis. The selected variables were primary site, T-stage, distances of the gross tumor volume (GTV) from parotid, RT technique, and surgery. Using propensity scores, the high RPLN spared group and treated group were matched with a 1:2 nearest-neighbor matching protocol with a caliper width of 0.3 standard deviations. A multivariate Cox proportional hazard model and Kaplan-Meier survival analysis were used to determine factors associated with recurrence outcomes. Statistical analyses were performed using $\mathrm{R}$ software (version 3.5.3; R Foundation for Statistical Computing, Vienna, Austria). Statistical significance was set at $p<0.05$.

\section{Results}

\section{Patient characteristics}

Table 1 summarized patient characteristics of the two groups according to high RPLNs treatment status. The median follow-up 
Table 1. Patients' characteristics in the entire cohort and propensity score matched cohort

\begin{tabular}{|c|c|c|c|c|c|c|}
\hline & \multicolumn{3}{|c|}{ Entire cohort (before matching) } & \multicolumn{3}{|c|}{ Matched cohort (after matching) } \\
\hline & $\begin{array}{l}\text { High-RPLNs spared } \\
(n=45)\end{array}$ & $\begin{array}{l}\text { High-RPLNs treated } \\
\quad(n=144)\end{array}$ & p-value & $\begin{array}{l}\text { High-RPLNs spared } \\
(n=23)\end{array}$ & $\begin{array}{l}\text { High-RPLNs treated } \\
(n=46)\end{array}$ & p-value \\
\hline Age (yr) & $65.4 \pm 9.2$ & $65.1 \pm 9.6$ & 0.865 & $64.6 \pm 10.6$ & 0.892 & 0.892 \\
\hline Follow-up duration (mo) & $59.6 \pm 22.9$ & $56.4 \pm 26.4$ & 0.456 & $57.7 \pm 27.8$ & 0.538 & 0.538 \\
\hline Gender & & & 0.575 & & 0.477 & 0.477 \\
\hline Male & $40(88.9)$ & $121(84.0)$ & & $38(82.6)$ & & \\
\hline Female & $5(11.1)$ & $23(16.0)$ & & $8(17.4)$ & & \\
\hline Smoking & & & 0.791 & & 0.304 & 0.304 \\
\hline Never smoker & $20(44.4)$ & $68(47.9)$ & & $25(54.3)$ & & \\
\hline Current smoker & $14(31.1)$ & $46(32.4)$ & & $15(32.6)$ & & \\
\hline Ex-smoker & $11(24.4)$ & $28(19.7)$ & & $6(13.0)$ & & \\
\hline HPV status & & & 0.981 & & 0.521 & 0.521 \\
\hline Negative & $10(22.2)$ & $34(23.6)$ & & $4(17.4)$ & & \\
\hline Positive & $25(55.6)$ & $79(54.9)$ & & $16(69.6)$ & & \\
\hline Unknown & $10(22.2)$ & $31(21.5)$ & & $3(13.0)$ & & \\
\hline p16 status & & & 0.632 & & 0.861 & 0.861 \\
\hline Negative & $6(13.3)$ & $26(18.1)$ & & $3(13.0)$ & & \\
\hline Positive & $30(66.7)$ & $96(66.7)$ & & $17(73.9)$ & & \\
\hline Unknown & $9(20.0)$ & $22(15.3)$ & & $3(13.0)$ & & \\
\hline Primary site & & & $<0.001$ & & 1 & 1 \\
\hline Pharyngeal wall & $1(2.2)$ & $1(0.7)$ & & $0(0)$ & & \\
\hline Soft palate & $2(4.4)$ & $6(4.2)$ & & $5(10.9)$ & & \\
\hline Tongue base & $16(35.6)$ & $8(5.6)$ & & $6(13.0)$ & & \\
\hline Tonsil & $24(53.3)$ & $129(89.6)$ & & $35(76.1)$ & & \\
\hline Vallecular & $2(4.4)$ & $0(0)$ & & $0(0)$ & & \\
\hline Clinical T stage (AJCC 7th) & & & 0.112 & & 0.744 & 0.744 \\
\hline 1 & $14(31.1)$ & $31(21.5)$ & & $12(26.1)$ & & \\
\hline 2 & $23(51.1)$ & $79(54.9)$ & & $23(50.0)$ & & \\
\hline 3 & $4(8.9)$ & 19 (13.2) & & $6(13.0)$ & & \\
\hline 4 & $4(8.9)$ & $15(10.4)$ & & $5(10.9)$ & & \\
\hline Clinical N stage (AJCC 7th) & & & 0.848 & & 0.717 & 0.717 \\
\hline 0 & $4(8.9)$ & $20(13.9)$ & & $9(19.6)$ & & \\
\hline 1 & $11(24.4)$ & $25(17.4)$ & & $4(8.7)$ & & \\
\hline $2 a$ & $2(4.4)$ & $7(4.9)$ & & $2(4.3)$ & & \\
\hline $2 b$ & $22(48.9)$ & $74(51.4)$ & & $25(54.3)$ & & \\
\hline $2 c$ & $6(13.3)$ & $17(11.8)$ & & $6(13.0)$ & & \\
\hline 3 & $0(0)$ & $1(0.7)$ & & $0(0)$ & & \\
\hline Prescribed dose (Gy) & $65.7 \pm 3.2$ & $66.3 \pm 2.6$ & 0.208 & $65.4 \pm 2.2$ & 0.82 & 0.820 \\
\hline Radiotherapy technique & & & 0.254 & & & \\
\hline 3D-CRT & $4(8.9)$ & $25(17.4)$ & & $0(0)$ & 1 & 1 \\
\hline IMRT & $41(91.1)$ & 119 (82.6) & & $46(100)$ & & \\
\hline Parotid to GTVpn distance $(\mathrm{cm})$ & & & 0.006 & & 0.943 & 0.943 \\
\hline$<0.5$ & $10(22.2)$ & $65(45.1)$ & & $8(17.4)$ & & \\
\hline$\geq 0.5$ and $<1$ & $17(37.8)$ & $42(29.2)$ & & $18(39.1)$ & & \\
\hline$\geq 1$ & $18(40.0)$ & $37(25.7)$ & & $20(43.5)$ & & \\
\hline
\end{tabular}

Values are presented as mean \pm standard deviation or number (\%).

RPLNs, retropharyngeal lymph nodes; HPV, human papillomavirus; AJCC, American Joint Committee on Cancer; 3D-CRT, three-dimensional conformal radiotherapy; IMRT, intensity-modulated radiotherapy; GTVpn, gross tumor volume for primary tumor mass and lymph node metastases. 
time was 66 months (range, 2 to 136 months), and the median age was 64 years (range, 43 to 90 years). Of the entire cohort, high RPLNs were treated in 144 (76.2\%) and spared in 45 (23.8\%). There were no significant between-group differences in age, sex, smoking, HPV/p16 status, and clinical T/N stages. The distances of the GTV from the parotid and anatomic subsites were significantly associated with the high RPLN treatment status. The high RPLNs of patients with tongue-base primary tumors were often spared because the tongue base is located relatively far from the high RPLN. After propensity score matching, a subset of 69 patients were chosen for matched pairs, which exhibiting similar baseline characteristics based on our propensity score model.

\section{Pattern of failure}

Of the 189 patients, 36 (19.0\%) showed recurrence after radiotherapy. The median time to failure was 11 months (range, 3 to 50 months). The failure patterns are shown in Fig. 1E. The incidences of local, regional, and distant failure were $9.0 \%, 10.1 \%$, and $5.8 \%$, respectively. Table 2 demonstrates the results of the univariate and multivariate Cox proportional hazard analyses for locoregional failure-free survival (LRFFS). Multivariate analysis revealed that old age was associated with poor prognosis, and HPV positivity was associated with a favorable prognosis. Kaplan-Meier analysis showed that high RPLN treatment was not significantly associated with LRFFS, and RPLN failure-free survival in the entire and the matched cohort (Fig. 1A-1D). When comparing the two groups for entire cohort, the 5-year rates of LRFFS, RPLN failure-free survival, distant metastasis-free survival, and overall survival were $82.8 \%$ and 90.6\% ( $p=0.14), 99.3 \%$ and 95.0\% ( $p=0.09$ ), 93.1\% and 93.3\% ( $p=0.98)$, and 85.7\% and 87.2\% ( $p=0.34)$ in the treated and spared groups, respectively.

Table 3 summarizes the characteristics of the patients who developed RPLN recurrence. One patient $(1 / 144,0.07 \%)$ presented with RPLN failure combined with local recurrence from palate to the skull base. Two $(2 / 45,4.4 \%)$ patients in the high RPLN-treated group developed RPLN recurrence; one of them had simultaneous lung metastasis and the other had RPLN failure with level II nodal failure. The patient with regional recurrence alone was successfully managed with stereotactic body radiation therapy (SBRT) to the RPLN area, level II neck dissection, and postoperative CCRT. No evidence of RPLNs recurrence until 22 months after SBRT. The other two patients died from disease progression.

\section{Ipsilateral parotid dose and xerostomia}

Table 4 shows the results of elective high RPLNs treatment. After matching, patients in the high RPLN-treated group had a signifi-
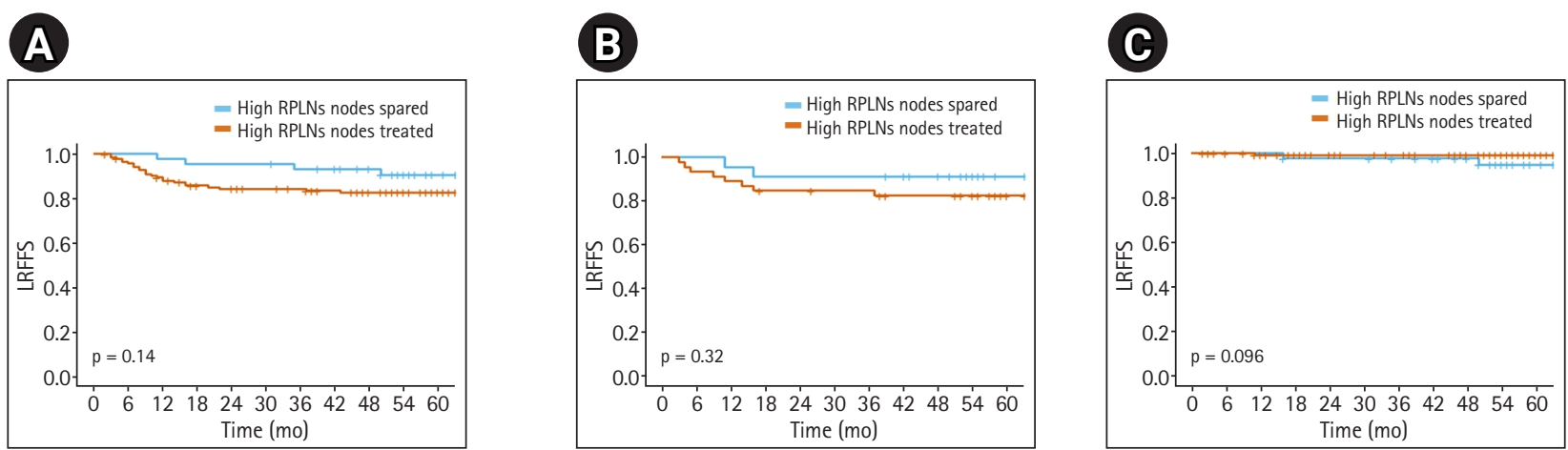

\section{(D)}
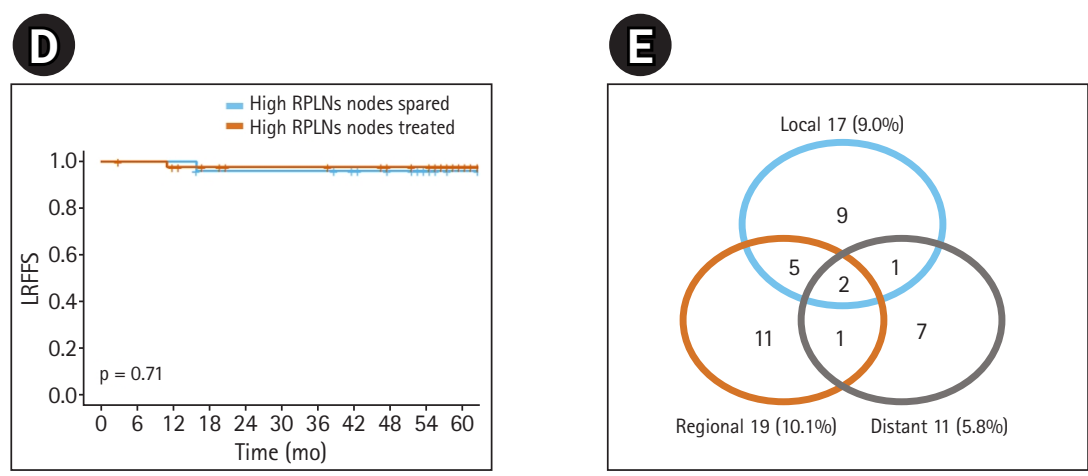

Fig. 1. Kaplan-Meier plot for clinical outcomes of ( $A, B)$ locoregional failure-free survival (LRFFS), and (C, D) retropharyngeal failure-free survival (RPFFS) according to high RPLNs treatment status in the entire cohort and in the matched cohort. (E) The distribution of first failure pattern. RPLNs: retropharyngeal lymph nodes. 
Table 2. Univariate and multivariate survival analysis for locoregional failure-free survival

\begin{tabular}{|c|c|c|c|c|c|c|}
\hline & \multicolumn{3}{|c|}{ Univariate } & \multicolumn{3}{|c|}{ Multivariate } \\
\hline & $\mathrm{HR}$ & $95 \% \mathrm{Cl}$ & $p$-value & $\mathrm{HR}$ & $95 \% \mathrm{Cl}$ & $p$-value \\
\hline Age & 1.07 & $1.03-1.11$ & 0.001 & 1.06 & $1.02-1.11$ & 0.003 \\
\hline Clinical T stage, $13 / 4$ & 2.02 & $0.94-4.35$ & 0.074 & 2.00 & $0.90-4.45$ & 0.088 \\
\hline \multicolumn{7}{|l|}{ Smoking } \\
\hline Never smoker & - & - & - & - & - & - \\
\hline Current smoker & 2.39 & $0.98-5.85$ & 0.056 & 2.14 & $0.87-5.29$ & 0.098 \\
\hline Ex-smoker & 2.82 & $1.09-7.33$ & 0.033 & 2.35 & $0.89-6.20$ & 0.085 \\
\hline \multicolumn{7}{|l|}{ HPV } \\
\hline Negative & - & - & - & - & - & - \\
\hline Positive & 0.31 & $0.13-0.71$ & 0.005 & 0.40 & $0.17-0.92$ & 0.032 \\
\hline Unknown & 0.47 & $0.18-1.25$ & 0.130 & 0.38 & $0.14-1.04$ & 0.061 \\
\hline
\end{tabular}

$\mathrm{HR}$, hazard ratio; $\mathrm{Cl}$, confidence interval; HPV, human papillomavirus.

Table 3. Demographics and treatment of patients with RPLN recurrences

\begin{tabular}{|c|c|c|c|}
\hline & Patient 1 & Patient 2 & Patient 3 \\
\hline Age (yr) & 76 & 63 & 70 \\
\hline Stage (AJCC 7th) & cT4N1M0 & $\mathrm{cT} 2 \mathrm{~N} 2 \mathrm{bM} 0$ & cT1N3M0 \\
\hline Smoking & Current & Never & Ex-smoker \\
\hline p16 & Negative & Positive & Positive \\
\hline \multicolumn{4}{|l|}{ Location } \\
\hline Primary & Soft palate & Tonsil & Tonsil \\
\hline High-RPLNs to GTV (cm) & $\leq 1$ & $\leq 1$ & $>1$ \\
\hline \multicolumn{4}{|l|}{ Treatment } \\
\hline Purpose & Radical & Radical & Radical \\
\hline High-RPLNs & Treated & Spared & Spared \\
\hline \multicolumn{4}{|l|}{ Recurrence } \\
\hline First failure & Ipsilateral palate to skull base, RPLN & Ipsilateral RPLN, nasopharynx, lung & Ipsilateral RPLN \\
\hline Disease-free interval (mo) & 11 & 16 & 50 \\
\hline
\end{tabular}

AJCC, American Joint Committee on Cancer; RPLNs, retropharyngeal lymph nodes; GTV, gross tumor volume.

cantly higher mean ipsilateral parotid dose than those in the high RPLN-spared group (29.9 Gy vs. 20.8 Gy; $p$ < 0.001). Significantly more patients in the high RPLN-spared group fulfilled the Quantitative Analyses of Normal Tissue Effects in the Clinic criteria for parotid gland dose constraints (unilateral parotid mean dose $<20$ Gy) than in the high RPLN-treated group (39.1\% vs. 6.5\%; $p<$ 0.001) [21]. Consequently, the incidence of chronic xerostomia at one year after radiotherapy was significantly lower in the high RPLN-spared group than in the high RPLN-treated group (no chronic xerostomia, $43.5 \%$ vs. $13.0 \% ; p=0.023$ ). Fig. 2 represents the dose distribution of the high RPLN-treated group and the high RPLN-spared group. Comparing two patients with similar clinical conditions, high RPLNs sparing seems to be an effective way of reducing radiation dose for bilateral parotid glands. High RPLNs spared patient had lower mean doses to ipsilateral parotid gland (17.7 Gy vs. $24.7 \mathrm{~Gy}$ ), and contralateral parotid gland (7.0 Gy vs.
$12.8 \mathrm{~Gy})$ than high RPLNs treated patient.

\section{Discussion and Conclusion}

OPC has been associated with a risk of RPLN metastasis, with RPLN involvement in approximately $10 \%-20 \%$ of patients [22-24]. Since RPLNs are anatomically difficult to approach, standard neck dissection does not include these nodes. Thus RPLN metastasis must be diagnosed based on radiographic findings alone, this makes it difficult to diagnose RPLN accurately [25]. These nodes can be effectively treated using CCRT. However, this may significantly compromise patients' $\mathrm{QOL}$, since these nodes are located proximal to radiosensitive structures such as the parotid gland, posterior pharyngeal wall, and pharyngeal constrictor muscles. Therefore, there is ongoing debate regarding the delineation of radiotherapy fields. According to consensus guidelines, the cranial border for RPLN 


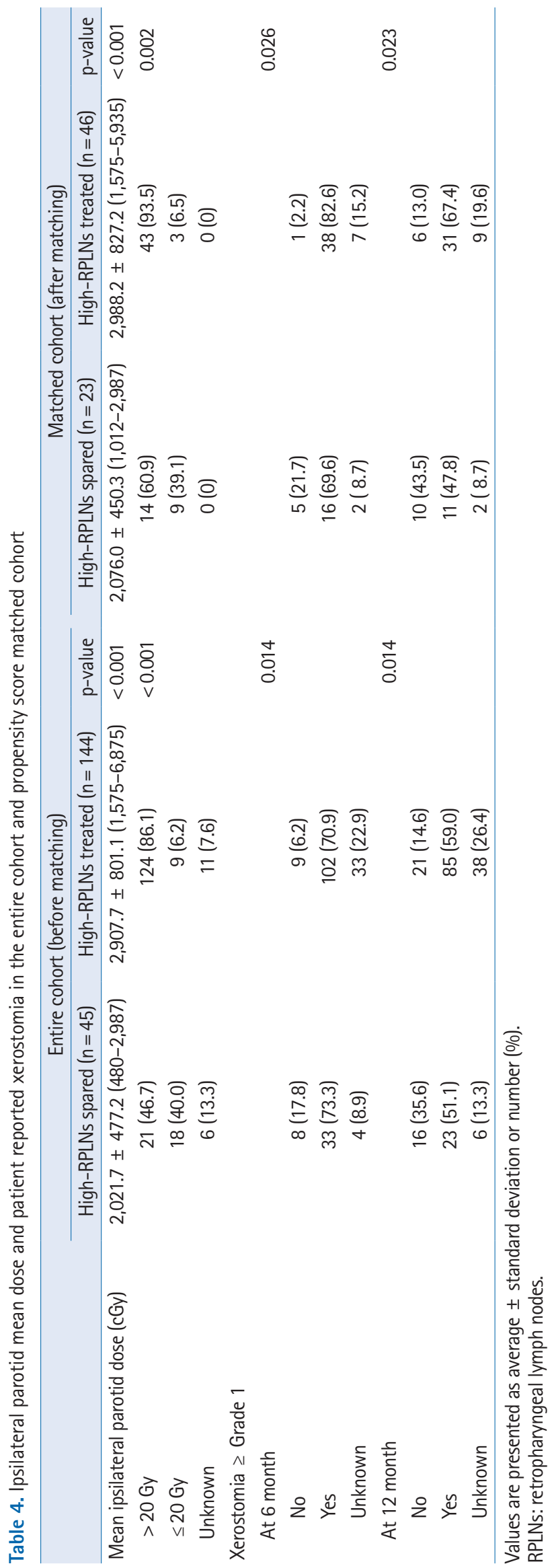

treatment is the upper edge of the $\mathrm{C} 1$ vertebral body/hard palate [13]. Unlike nasopharyngeal cancer, most OPCs are located inferior to the lateral process of the $\mathrm{C} 1$ vertebra. Due to their anatomic location, metastasis to a high RPLN requires retrograde lymphatic flow from the OPC, and metastases to these nodes are considered rare. Tang et al. [23] showed that RPLN metastasis is associated with increased nodal burden. None of our patients with single node positivity exhibited RPLN metastasis. This suggests that RPLN metastasis occurs late in the disease course, and the treatment of RPLN should be individualized based on each patient's risk level.

Due to the excellent prognosis of HPV-induced OPC, there has been an increasing interest in the QOL of patients with OPC $[1,12]$. Currently, treatment de-escalation, which can be achieved by lowering the dose or reducing the field of radiation, has received attention. Since Eisbruch et al. [26] reported three marginal RPLN failures in 80 patients with $\mathrm{OPC}$, it has been recommended that RPLNs be treated bilaterally. However, in their prospective study, Spencer et al. [16] showed that sparing the contralateral RPLN and high level II nodes is safe and improves the QOL of patients with head and neck cancer. There is consensus regarding contralateral high RPLN sparing $[17,18]$. However, the safety of sparing ipsilateral RPLNs has not been sufficiently evaluated. Previously, Kjems et al. [19] reported that ipsilateral RPLNs were excluded from the elective target volume in 469 patients with OPC without posterior pharyngeal wall involvement; only one of them developed RPLN recurrence. In that study, the CTV encompassed the GTV with an additional 14-mm margin, and ipsilateral RPLNs were not included in the CTV unless the RPLN areas were close to the GTV or the posterior pharyngeal wall was involved.

In our study, three RPLN recurrences (1.6\%) occurred among 189 patients with OPC who showed no evidence of RPLN metastasis at initial diagnosis. Of the three patients with recurrence, the RPLNs of one $(1 / 144,0.07 \%)$ was treated and those of the others $(2 / 45$, 4.4\%) were untreated. One patient in the high RPLN-spared group developed RPLN recurrence with pulmonary lymphangitic metastasis. In this patient, there was a short distance $(\leq 1 \mathrm{~cm})$ from the primary GTV to the high RPLN. Retrospectively, we believe this patient would have benefited from high RPLN treatment. Another patient in the high RPLN-spared group developed RPLN recurrence and subsequent level II nodal failure at 50 and 62 months after RT, respectively. This patient underwent SBRT to treat isolated RPLN recurrence. After SBRT, there was no evidence of RPLN recurrence until the last follow-up. However, this patient developed level II recurrence with extracapsular extension and underwent modified radical neck dissection and postoperative CCRT without RPLN treatment. Currently, there is no evidence of recurrence or severe toxicity after the third course of RT. Historically, RPLN recurrence 

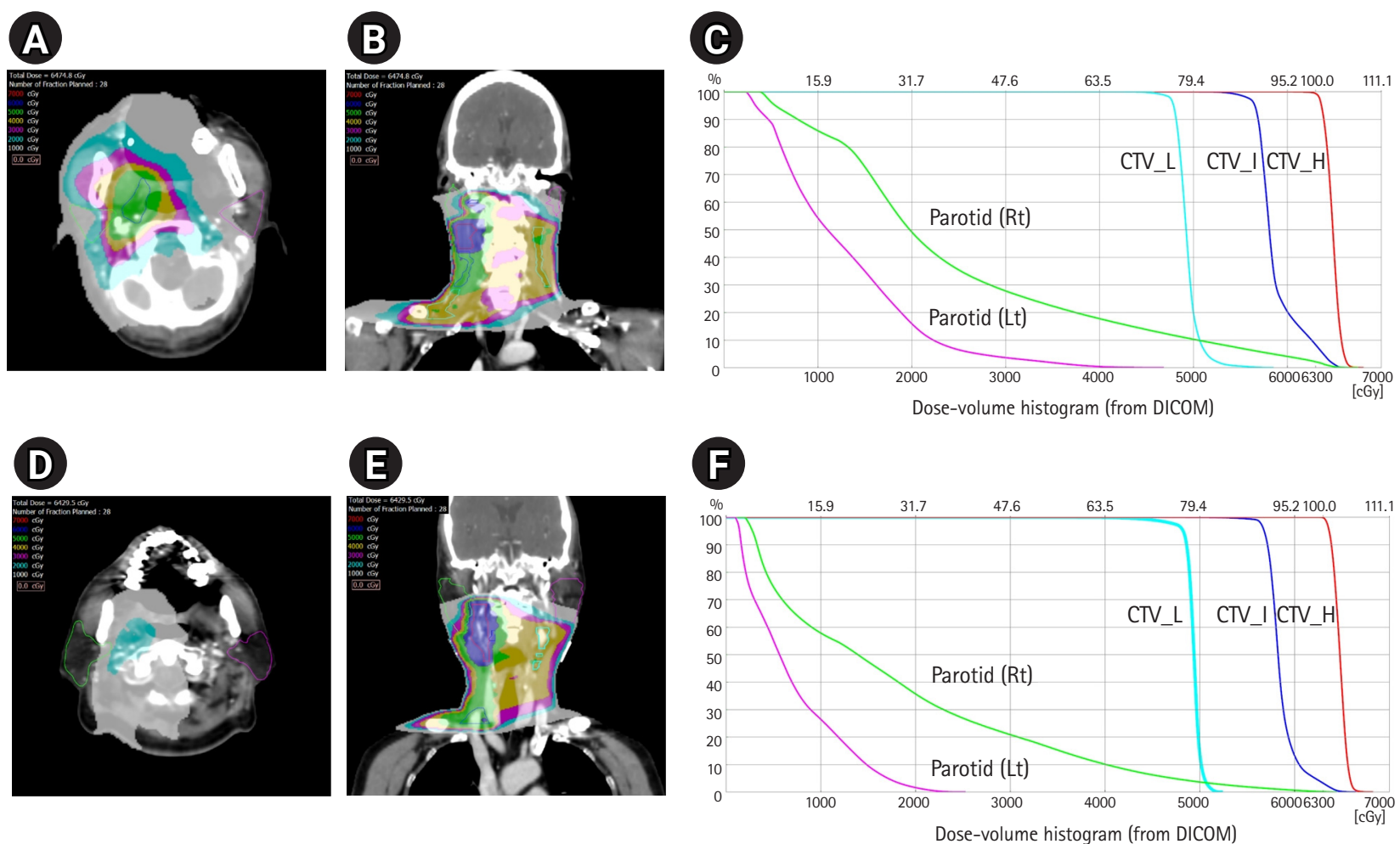

Fig. 2. Dose distribution comparison of adjuvant radiotherapy plans using volume modulated arc therapy technique for two tonsil cancer patients staged pT1N2bM0, who were (A-C) treated for high RPLNs (retropharyngeal lymph nodes) and (D-F) spared for high RPLNs. CTV, clinical target volume.

was considered difficult to salvage. Modern radiotherapy techniques make it possible for RPLN re-irradiation with tolerable toxicity and good local control $[27,28]$. However, treatment for RPLN failure is still challenging because of its close proximity to critical structures such as the carotid artery, and careful patient selection is necessary to ensure safe outcomes following RPLN sparing $[22,28,29]$.

Here, we present evidence that RPLN recurrence is rare, and careful sparing of the RPLN helps reduce chronic xerostomia in patients with OPC. Patients in the high RPLN-spared group had a lower ipsilateral parotid dose and, consequently, less chronic xerostomia at 1 year than those in the high RPLN-treated group in entire cohort (grade $0,35.6 \%$ vs. 14.6\%; $p=0.014$ ) and matched cohort (grade $0,43.5 \%$ vs. $13.0 \% ; p=0.023$ ). As sparing of the high RPLN could result in more RPLN recurrences in some settings, the high RPLNs should be treated in patients with risk factors such as posterior pharyngeal wall involvement, bulky nodal burden, and primary tumor mass near the high RPLNs. Further studies are needed to identify the risk factors for RPLN recurrence for ensuring the safety of high RPLN sparing.

\section{Conflict of Interest}

No potential conflict of interest relevant to this article was reported.

\section{References}

1. Chaturvedi AK, Engels EA, Pfeiffer RM, et al. Human papillomavirus and rising oropharyngeal cancer incidence in the United States. J Clin Oncol 2011;29:4294-301.

2. Guo T, Eisele DW, Fakhry C. The potential impact of prophylactic human papillomavirus vaccination on oropharyngeal cancer. Cancer 2016;122:2313-23.

3. You EL, Henry M, Zeitouni AG. Human papillomavirus-associated oropharyngeal cancer: review of current evidence and management. Curr Oncol 2019;26:119-23.

4. Ang KK, Harris J, Wheeler R, et al. Human papillomavirus and survival of patients with oropharyngeal cancer. N Engl J Med 2010;363:24-35.

5. Elrefaey S, Massaro MA, Chiocca S, Chiesa F, Ansarin M. HPV in oropharyngeal cancer: the basics to know in clinical practice. Acta Otorhinolaryngol Ital 2014;34:299-309. 
6. O'Sullivan B, Huang SH, Su J, et al. Development and validation of a staging system for HPV-related oropharyngeal cancer by the International Collaboration on Oropharyngeal cancer Network for Staging (ICON-S): a multicentre cohort study. Lancet Oncol 2016;17:440-51.

7. Pignon JP, le Maitre A, Maillard E, Bourhis J; MACH-NC Collaborative Group. Meta-analysis of chemotherapy in head and neck cancer (MACH-NC): an update on 93 randomised trials and 17,346 patients. Radiother Oncol 2009;92:4-14.

8. Gunn GB, Debnam JM, Fuller CD, et al. The impact of radiographic retropharyngeal adenopathy in oropharyngeal cancer. Cancer 2013;119:3162-9.

9. Hoxbroe Michaelsen S, Gronhoj C, Hoxbroe Michaelsen J, Friborg J, von Buchwald C. Quality of life in survivors of oropharyngeal cancer: a systematic review and meta-analysis of 1366 patients. Eur J Cancer 2017;78:91-102.

10. Mehanna H, Robinson M, Hartley A, et al. Radiotherapy plus cisplatin or cetuximab in low-risk human papillomavirus-positive oropharyngeal cancer (De-ESCALaTE HPV): an open-label randomised controlled phase 3 trial. Lancet 2019;393:51-60.

11. Gillison ML, Trotti AM, Harris J, et al. Radiotherapy plus cetuximab or cisplatin in human papillomavirus-positive oropharyngeal cancer (NRG Oncology RTOG 1016): a randomised, multicentre, non-inferiority trial. Lancet 2019;393:40-50.

12. Adelstein DJ, Ismaila N, Ku JA, et al. Role of treatment deintensification in the management of p16+ oropharyngeal cancer: ASCO provisional clinical opinion. J Clin Oncol 2019;37:1578-89.

13. Gregoire V, Ang K, Budach W, et al. Delineation of the neck node levels for head and neck tumors: a 2013 update. DAHANCA, EORTC, HKNPCSG, NCIC CTG, NCRI, RTOG, TROG consensus guidelines. Radiother Oncol 2014;110:172-81.

14. Deasy JO, Moiseenko V, Marks L, Chao KS, Nam J, Eisbruch A. Radiotherapy dose-volume effects on salivary gland function. Int J Radiat Oncol Biol Phys 2010;76(3 Suppl):S58-63.

15. Nutting CM, Morden JP, Harrington KJ, et al. Parotid-sparing intensity modulated versus conventional radiotherapy in head and neck cancer (PARSPORT): a phase 3 multicentre randomised controlled trial. Lancet Oncol 2011;12:127-36.

16. Spencer $\mathrm{CR}$, Gay $\mathrm{HA}$, Haughey $\mathrm{BH}$, et al. Eliminating radiotherapy to the contralateral retropharyngeal and high level II lymph nodes in head and neck squamous cell carcinoma is safe and improves quality of life. Cancer 2014;120:3994-4002.

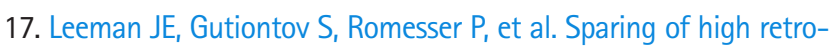
pharyngeal nodal basins in patients with unilateral oropharyngeal carcinoma treated with intensity modulated radiation ther- apy. Pract Radiat Oncol 2017;7:254-9.

18. Nagatsuka M, Hughes RT, Shenker RF, Frizzell BA, Greven KM. Omitting elective irradiation of the contralateral retropharyngeal nodes in oropharyngeal squamous cell carcinoma treated with intensity-modulated radiotherapy. Cureus 2019;11:e3825.

19. Kjems J, Gothelf AB, Hakansson K, Specht L, Kristensen CA, Friborg J. Elective nodal irradiation and patterns of failure in head and neck cancer after primary radiation therapy. Int J Radiat Oncol Biol Phys 2016;94:775-82.

20. Cox JD, Stetz J, Pajak TF. Toxicity criteria of the Radiation Therapy Oncology Group (RTOG) and the European Organization for Research and Treatment of Cancer (EORTC). Int J Radiat Oncol Biol Phys 1995;31:1341-6.

21. Marks LB, Yorke ED, Jackson A, et al. Use of normal tissue complication probability models in the clinic. Int J Radiat Oncol Biol Phys 2010;76(3 Suppl):S10-9.

22. Gross ND, Ellingson TW, Wax MK, Cohen Jl, Andersen PE. Impact of retropharyngeal lymph node metastasis in head and neck squamous cell carcinoma. Arch Otolaryngol Head Neck Surg 2004;130:169-73.

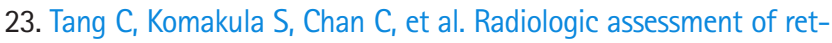
ropharyngeal node involvement in oropharyngeal carcinomas stratified by HPV status. Radiother Oncol 2013;109:293-6.

24. Dirix P, Nuyts $S$, Bussels B, Hermans R, Van den Bogaert W. Prognostic influence of retropharyngeal lymph node metastasis in squamous cell carcinoma of the oropharynx. Int J Radiat Oncol Biol Phys 2006;65:739-44.

25. Chung EJ, Oh Jl, Choi KY, et al. Pattern of cervical lymph node metastasis in tonsil cancer: predictive factor analysis of contralateral and retropharyngeal lymph node metastasis. Oral Oncol 2011;47:758-62.

26. Eisbruch $A$, Marsh LH, Dawson LA, et al. Recurrences near base of skull after IMRT for head-and-neck cancer: implications for target delineation in high neck and for parotid gland sparing. Int J Radiat Oncol Biol Phys 2004;59:28-42.

27. Vargo JA, Ward MC, Caudell JJ, et al. A multi-institutional comparison of SBRT and IMRT for definitive reirradiation of recurrent or second primary head and neck cancer. Int J Radiat Oncol Biol Phys 2018;100:595-605.

28. Pollard C 3rd, Nguyen TP, Ng SP, et al. Clinical outcomes after local field conformal reirradiation of patients with retropharyngeal nodal metastasis. Head Neck 2017;39:2079-87.

29. Chan JY, Chow VL, Wong ST, Wei WI. Surgical salvage for recurrent retropharyngeal lymph node metastasis in nasopharyngeal carcinoma. Head Neck 2013;35:1726-31. 